M. Hara

Nagoya Math. J.

Vol. 49 (1973), 143-148

\title{
ON THE EXISTENCE OF VARIOUS BOUNDED HARMONIC FUNCTIONS WITH GIVEN PERIODS
}

\author{
MASARU HARA
}

1. Consider a pair $(R, \Gamma)$ of a Riemann surface $R$ and a period $\Gamma$. By a period $\Gamma$ we mean a real-valued function $\Gamma(\gamma)$ on one-dimensional cycles $\{\gamma\}$ of the Riemann surface $R$. Let $O_{X}^{*}$ be the class of pairs $(R, \Gamma)$ such that there is no harmonic function on the Riemann surface $R$ which satisfies a boundedness property $X$ and

$$
\int_{r}^{*} d u=\Gamma(\gamma)
$$

for every cycle $\gamma$. As for $X$ we let $B$ stand for boundedness, $D$ for the finiteness of the Dirichlet integral, $B D$ for $B$ and $D$. The relations to standard notations $\mathrm{O}_{A X}$ in the classification theory of Riemann surfaces (cf. [1]) should be clear. For example, $R \in O_{A D}$ means that $\left(R, \Gamma_{0}\right) \in O_{D}^{*}$, where $\Gamma_{0}(\gamma)=0$ for every cycle $\gamma$, and $R \in O_{A B D}$ means that $\left(R, \Gamma_{0}\right) \in O_{B D}^{*}$. From our standpoint H. Widom's articles [3] and [4] may be considered as the study of the class $O_{B}^{*}$. Our study may be also be considered as being in the frame work of that of Riemann matrices.

The well known Virtanen identity $O_{H D}=O_{H B D}$ is one of the beautiful results in the classification theory; what's more, the space $H B D(R)$ is dense in $H D(R)$ in the $C D$-topology (cf. [1, p. 178]). Therefore there exists a sequence $\left\{u_{n}\right\}$ in $H B D(R)$ convergent to a given $u \in H D(R)$ so that $\int_{r} * d u_{n}$ converges to $\int_{r} * d u$ for every cycle $\gamma$. In this connection one naturally asks whether $O_{D}^{*}=O_{B D}^{*}$. The question also relates to the unsettled strictness question $O_{A D} \subset O_{A B D}$. The main result of this paper is the following strict inclusion:

THEOREM.

$$
O_{D}^{*}<O_{B D}^{*}
$$

We will show that there exists a planar region $\Omega^{*}$ such that there Received May 19, 1972. 
exist $H D$-functions on $\Omega^{*}$ which have the same period as the given $H B$ functions on $\Omega^{*}$ but there exists no $H B$-function on $\Omega^{*}$ which has the same period as some $H D$-function on $\Omega^{*}$.

2. Let $\Omega$ denote the right half plane of the complex plane and $\Omega[a b]$ the right half plane less the interval $[a b]$ on the real axis. The function

$$
g\left(z, z_{0}\right)=\log \left|\frac{z+\bar{z}_{0}}{z-\bar{z}_{0}}\right|
$$

is the Green's function for the region $\Omega$ with pole at $z_{0}$. The function

$$
u[a b](\mathrm{z})=\int_{a}^{b} \log \left|\frac{z+t}{z-t}\right| d t \quad(0<a<b)
$$

is the potential whose support is the interval $[a b]$. Therefore $u[a b](z)$ is positive and harmonic on the region $\Omega[a b]$ and vanishes on the imaginary axis, and furthermore has the following properties:

LEMMA 1. Let $\beta$ be a simple curve oriented clockwise enclosing the interval $[a b]$. Then $u[a b]$ is continuous on the region $\Omega$ and

$$
\int_{\beta}^{*} d u[a b]=2 \pi(b-a)
$$

$$
\begin{aligned}
D(u[a b])= & \pi\left\{(2 b)^{2} \log 2 b-2(a+b)^{2} \log (a+b)+(2 a)^{2} \log 2 a\right\} \\
& +2 \pi(b-a)^{2} \log \frac{1}{b-a} .
\end{aligned}
$$

Proof. Put $u=u[a b]$. For $a \leq x \leq b$,

$$
\begin{aligned}
u(x)= & \int_{a}^{b} \log \left|\frac{x+t}{x-t}\right| d t \\
= & \int_{a}^{b} \log (x+t) d t-\int_{a}^{x} \log (x-t) d t-\int_{x}^{b} \log (t-x) d t \\
= & (x+b) \log (x+b)-(x+a) \log (x+a) \\
& -(x-a) \log (x-a)-(b-x) \log (b-x) .
\end{aligned}
$$

Thus $u(x)$ is continuous on the interval $[a b]$ which is the support of potential $u$, and therefore it follows from the continuity principle (cf. [2, p. 54]) that $u$ is continuous on the region $\Omega$.

Fix $x, a<x<b$, and consider 


$$
f(z)=\int_{a}^{b} \log \frac{z+t}{z-t} d t
$$

on the upper plane. Observe that

$$
f^{\prime}(z)=\int_{a}^{b}\left(\frac{1}{z+t}-\frac{1}{z-t}\right) d t
$$

Since

$$
\lim _{z \rightarrow x} \operatorname{Im}\left(\int_{a}^{b} \frac{1}{z+t} d t\right)=0
$$

and

$$
\int_{a}^{b} \frac{1}{t-z} d t=\log (b-z)-\log (a-z)
$$

whose imaginary part is the angle formed by the lines $\overline{z a}$ and $\overline{z b}$, we conclude that

$$
\lim _{z \rightarrow x} \operatorname{Im}\left(f^{\prime}(z)\right)=\pi .
$$

From this it follows that $* d u=\pi$ on the interval $(a b)$ considered as the degenerate closed curve traced in the negative direction.

Therefore (1) is trivially true. By

$$
D(u)=2 \pi \int_{a}^{b} u(t) d t
$$

and direct calculations, we obtain (2).

COROLlaRY. For $a \geq e$,

$$
\begin{gathered}
\int_{\beta} * d u[a a+1]=2 \pi ; \\
D(u[a a+1]) \leq 10 \pi \log a .
\end{gathered}
$$

Proof. The relation (3) is trivial and (4) is seen by direct calculations.

3. We denote by $D_{\mathrm{c}}$ the interior of the ellipse, whose horizontal axis is of length $\frac{1}{2}((1 / r)+r)=c$ and vertical axis $\frac{1}{2}((1 / r)-r)(0<r<1)$, less the interval with length 1 in the center on the horizontal axis. Let 
$v_{c}$ denote the harmonic measure of the interval with respect to the region $D_{c}$.

LEMMA 2. Let $\beta$ be a simple curve oriented clockwise enclosing the interval. Then

$$
\int_{\beta}^{*} d v_{c} \leq 2 \pi(\log c)^{-1}
$$

Proof. Suppose that the center of the ellipse is the origin. The function $z=\frac{1}{4}((1 / w)+w)$ maps the annulus $\{r<|w|<1\}$ conformally onto $D_{c}$, the circle $|w|=r$ onto the ellipse and the circle $|w|=1$ onto the interval. The harmonic measure of the circle $\{|w|=1\}$ with respect to the annulus $\{r<|w|<1\}$ is the function

$$
\log \frac{|w|}{r} / \log \frac{1}{r}
$$

whose flux is $2 \pi(\log 1 / r)^{-1}$. Therefore

$$
\int_{\beta} * d v_{c}=2 \pi\left(\log \frac{1}{r}\right)^{-1}=2 \pi\left(\log \left(c+\left(c^{2}-1\right)^{\frac{1}{2}}\right)\right)^{-1} \leq 2 \pi(\log 2 c)^{-1} .
$$

4. Put

$$
a_{n}=\exp \left(\sum_{k=0}^{n} 2^{k}\right)
$$

and

$$
\Omega^{*}=\bigcap_{n=1}^{\infty} \Omega\left[a_{n} a_{n}+1\right]
$$

and $u_{n}=u\left[a_{n} a_{n}+1\right]$ and $u=\sum_{n=1}^{\infty} n 2^{-n} u_{n}$. Let $\gamma_{n}$ be a simple curve oriented clockwise enclosing $\left[a_{n} a_{n}+1\right]$ so that $\gamma_{m}$ and $\gamma_{n}$ are disjoint if $m \neq n$. Then $\left\{\gamma_{n}\right\}_{n=1}^{\infty}$ is a homology basis of $\Omega^{*}$.

In order to prove our theorem it is sufficient to show the following lemma :

LEMMA 3. The region $\Omega^{*}$ has the following properties:

(i) The function $u$ belongs to $H D\left(\Omega^{*}\right)$;

(ii) No function belong to $H B\left(\Omega^{*}\right)$ has the same period as the function $u$;

(iii) Give any function $v$ belonging to $H B\left(\Omega^{*}\right)$, 


$$
v^{*}=\frac{1}{2 \pi} \sum_{n=1}^{\infty}\left(\int_{7 n} * d v\right) u_{n}
$$

belongs to $H D\left(\Omega^{*}\right)$ and has the same period as the function $v$.

Proof. Since

$$
\begin{aligned}
& D\left(u_{n}\right) \leq 10 \pi \log a_{n}=10 \pi \sum_{k=0}^{n} 2^{k} \leq 20 \pi 2^{n}, \\
& \sum_{n=0}^{\infty} n 2^{-n}\left(D\left(u_{n}\right)\right)^{\frac{1}{2}} \leq(20 \pi)^{\frac{1}{2}} n\left(2^{-\frac{1}{2}}\right)^{n}<\infty .
\end{aligned}
$$

Noticing this and using properties of $C D$-topology [1, p. 149], the function $u$ belongs to the class $H D\left(\Omega^{*}\right)$, i.e. (i) is true.

To prove (ii) it suffices to show that

$$
\lim _{n \rightarrow \infty} \frac{\int_{\gamma_{n}} * d v}{\int_{\gamma_{n}} * d u}=0
$$

for every $v \in H B\left(\Omega^{*}\right)$. We may, without loss of generality, assume that $M-1>v>1$. Let $D_{n}$ denote the region $D_{c}, c=a_{n}-a_{n-1}-\frac{1}{2}$, whose outer boundary is an ellipse having the center at $a_{n}+\frac{1}{2}$ and passing $a_{n-1}+1$, and let $v_{n}$ denote $2 M v_{c}$. For $\frac{1}{2}<t<1$, the set $\left\{z \in D_{n} ; t v_{n}>v\right\}$ contains a neighbourhood of the interval $\left[a_{n} a_{n}+1\right]$ and does not contain a neighbourhood of the ellipse. By the maximum principle, this set is a region and we can choose some $t$ so that the set $\left\{z \in D_{n} \mid t v_{n}=v\right\}$ is a simple regular closed curve, which is denoted by $\delta_{n}$, homologous to $\gamma_{n}$. Since

$$
\int_{\delta_{n}} * d t v_{n}>\int_{\delta_{n}} * d v
$$

and

$$
\int_{r_{n}} * d v_{n}=\int_{\hat{o}_{n}} * d v_{n}>t \int_{\delta_{n}} * d v_{n}=\int_{\delta_{n}} * d t v_{n}>\int_{\hat{\delta}_{n}} * d v=\int_{\gamma_{n}} * d v
$$

By Lemma 2,

$$
\begin{aligned}
0 & <\int * d v_{n} \leq 2 \pi M\left(\log \left(\left(a_{n}-a_{n-1}\right)-1 / 2\right)\right)^{-1} \\
& \leq 2 \pi M\left(\log \frac{a_{n}}{a_{n-1}}\right)^{-1}=2 \pi M 2^{-n}
\end{aligned}
$$


From

$$
\int_{\tau_{n}} * d u=n 2^{-n} \int_{\tau_{n}} * d u_{n}=2 \pi n 2^{-n},
$$

it follows that

$$
\int_{\gamma_{n}} * d u>\frac{1}{M} n \int_{r_{n}} * d v_{n}>\frac{1}{M} n \int_{\gamma_{n}} * d v
$$

Since $M-1>M-v>1$, by the same arguments,

$$
\int_{\gamma_{n}} * d u \geq-\frac{1}{M} n \int_{r_{n}} * d v
$$

The proof of (ii) is herewith complete.

Since $\int_{\gamma_{n}} * d v=o\left(n 2^{-n}\right)$, by the same argument as for the function $u$, we can show that the function $v^{*}$ belongs to $H D\left(\Omega^{*}\right)$. It is trivial that the function $v^{*}$ has the same period as the function $v$, and (iii) is obtained.

\section{REFERENCES}

[1] Sario, L. and M. Nakai: Classification Theory of Riemann Surfaces. Springer (1970).

[2] Tsuji, M.: Potential Theory in modern Function Theory. Maruzen (1959).

[3] Widom, H.: The maximum principle for multivalued analytic functions. Acta Math. 126 (1971), 63-82.

[4] ——: $\Omega_{p}$ sections of vector bundles over Riemann surfaces. Ann. of Math. 94 (1971), 304-324.

Applied physics, Faculty of Engineering

Nagoya University 